International Journal of Pure and Applied Mathematics

Volume 97 No. 4 2014, 491-495

ISSN: 1311-8080 (printed version); ISSN: 1314-3395 (on-line version)

url: http://www.ijpam.eu

doi: http://dx.doi.org/10.12732/ijpam.v97i4.8

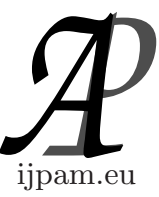

\title{
APPROXIMATION OF BINOMIAL DISTRIBUTION BY AN IMPROVED POISSON DISTRIBUTION
}

\author{
K. Teerapabolarn ${ }^{1}$, K. Jaioun ${ }^{2}$ \\ ${ }^{1,2}$ Department of Mathematics \\ Faculty of Science \\ Burapha University \\ Chonburi, 20131, THAILAND
}

\begin{abstract}
This paper derives an approximation of the binomial distribution with parameters $n$ and $p$ by an improved Poisson distribution with mean $\lambda=n p$. The approximation is more accurate than the Poisson approximation when $n$ is sufficiently large.
\end{abstract}

AMS Subject Classification: 62E17, 60F05

Key Words: binomial distribution, Poisson approximation, Poisson distribution

\section{Introduction}

It is well-known that a discrete random variable $X$ has the binomial distribution with parameters $n \in \mathbb{N}$ and $p \in(0,1)$ if and only if its probability function is of the form

$$
\mathbf{b}_{n, p}(x)=\left(\begin{array}{l}
n \\
x
\end{array}\right) p^{x} q^{n-x}, x=0,1, \ldots, n,
$$

where $E(X)=\frac{n q}{p}$ and variance $\operatorname{Var}(X)=\frac{n q}{p^{2}}$ are the mean and variance of this random variable, respectively. We known that if $n \rightarrow \infty$ and $p \rightarrow 0$ while $\lambda=n p$ remains fixed, then $\mathbf{b}_{n, p}(x) \rightarrow \mathbf{p}_{\lambda}(x)=\frac{e^{-\lambda} \lambda^{x}}{x !}$ for every $x \in$

Received: July 2, 2014

(c) 2014 Academic Publications, Ltd.

$\S$ Correspondence author url: www.acadpubl.eu 
$\{0,1, \ldots, n\}$. Therefore, the Poisson distribution with mean $\lambda=n p$ can be used as an approximation of the binomial distribution when $n$ is large and $p$ is small. In this case, there has been much methodological research related to the Poisson approximation to the binomial distribution. For example, [1] gave some inequalities of binomial and Poisson probabilities, [4] and [6] gave inequalities for the ratio of Poisson and binomial probabilities and in terms of the relative error of two such probabilities can be found in [3], [2] and [7] [10]. However, there has no research related to improved Poisson approximation to the binomial distribution. In this paper, we are interested to derive an improved Poisson probability function, $\widehat{\mathbf{p}}_{\lambda}(x)$, for approximating the binomial probability function. The accuracy of the approximation is measured in the form of $\left|\mathbf{b}_{n, p}(x)-\widehat{\mathbf{p}}_{\lambda}(x)\right|$ for $x \in\{0,1, \ldots, n\}$. The result of this study is in Section 2. In Section 3, some numerical examples are given to illustrate the improved approximation and the conclusion of this study is presented in the last section.

\section{Result}

We use the same method in [5] to derive an improved Poisson distribution that can be obtained from the binomial distribution. The following lemma follows from [5].

Lemma 2.1. For $x, n \in \mathbb{N}$, then the following property holds:

$$
\prod_{i=0}^{x-1}\left(1-\frac{i}{n}\right)=\frac{1}{1+\frac{x(x-1)}{2 n}+O\left(\frac{1}{n^{2}}\right)} .
$$

Theorem 2.1. For $x \in\{0,1, \ldots, n\}$ and $\lambda=n p$, if $n$ is large then we have the following:

$$
\mathbf{b}_{n, p}(x) \approx \widehat{\mathbf{p}}_{\lambda}(x)
$$

where $\widehat{\mathbf{p}}_{\lambda}(x)=\frac{\mathbf{p}_{\lambda}(x)}{e^{\frac{\lambda^{2}}{2 n}} q^{x}\left\{1+\frac{x(x-1)}{2 n}\right\}}$.

Proof. For $x=0, \mathbf{b}_{n, p}(0)=(1-p)^{n}=\left(1-\frac{\lambda}{n}\right)^{n}=e^{n \log \left(1-\frac{\lambda}{n}\right)}=e^{-\lambda-\frac{\lambda^{2}}{2 n}-\cdots}$. Thus, when $n$ is large, we have $\mathbf{b}_{n, p}(0)=\frac{e^{-\lambda}}{e^{\frac{\lambda}{2 n}}}=\frac{\mathbf{p}_{\lambda}(0)}{e^{\frac{\lambda^{2}}{2 n}}}$.

For $x \in\{1, \ldots, n\}$,

$$
\mathbf{b}_{n, p}(x)=\frac{n !}{x !(n-x) !} \frac{(n p)^{x} q^{n}}{n^{x} q^{x}}
$$




$$
\begin{aligned}
& =\frac{\lambda^{x}}{x ! q^{x}}\left[\frac{n}{n} \cdots\left(1-\frac{x-1}{n}\right)\right]\left(1-\frac{\lambda}{n}\right)^{n} \\
& =\frac{\lambda^{x}}{x ! q^{x}} \prod_{i=0}^{x-1}\left(1-\frac{i}{n}\right)\left(e^{-\lambda-\frac{\lambda^{2}}{2 n}-\cdots}\right) \\
& =\frac{\mathbf{p}_{\lambda}(x) e^{-\frac{\lambda^{2}}{2 n}-\cdots}}{q^{x}\left\{1+\frac{x(x-1)}{2 n}+O\left(\frac{1}{n^{2}}\right)\right\}} \text { (by Lemma 2.1). }
\end{aligned}
$$

For large $n$, we obtain

$$
\mathbf{b}_{n, p}(x) \approx \frac{\mathbf{p}_{\lambda}(x)}{e^{\frac{\lambda^{2}}{2 n}} q^{x}\left\{1+\frac{x(x-1)}{2 n}\right\}},
$$

which yields (2.2).

\section{Numerical Examples}

The following examples have been given to illustrate how well an improved Poisson distribution approximates a binomial distribution.

3.1. Let $n=100$ and $p=0.01$, then $\lambda=1.0$ and the numerical results are as follows:

\begin{tabular}{cccccc}
\hline$x$ & $\mathbf{b}_{n, p}(x)$ & $\widehat{\mathbf{p}}_{\lambda}(x)$ & $\mathbf{p}_{\lambda}(x)$ & $\left|\mathbf{b}_{n, p}(x)-\widehat{\mathbf{p}}_{\lambda}(x)\right|$ & $\left|\mathbf{b}_{n, p}(x)-\mathbf{p}_{\lambda}(x)\right|$ \\
\hline 0 & 0.36603234 & 0.36604463 & 0.36787944 & 0.00001229 & 0.00184710 \\
1 & 0.36972964 & 0.36974206 & 0.36787944 & 0.00001242 & 0.00185020 \\
2 & 0.18486482 & 0.18488952 & 0.18393972 & 0.00002470 & 0.00092510 \\
3 & 0.06099917 & 0.06104358 & 0.06131324 & 0.00004441 & 0.00031407 \\
4 & 0.01494171 & 0.01497877 & 0.01532831 & 0.00003706 & 0.00038660 \\
5 & 0.00289779 & 0.00291598 & 0.00306566 & 0.00001819 & 0.00016787 \\
6 & 0.00046345 & 0.00046956 & 0.00051094 & 0.00000611 & 0.00004749 \\
7 & 0.00006286 & 0.00006440 & 0.00007299 & 0.00000153 & 0.00001013 \\
8 & 0.00000738 & 0.00000769 & 0.00000912 & 0.00000030 & 0.00000174 \\
9 & 0.00000076 & 0.00000081 & 0.00000101 & 0.00000005 & 0.00000025 \\
10 & 0.00000007 & 0.00000008 & 0.00000010 & 0.00000001 & 0.00000003 \\
\hline
\end{tabular}

3.2. Let $n=1000$ and $p=0.005$, then $\lambda=5.0$ and the numerical results are as follows: 


\begin{tabular}{cccccc}
\hline$x$ & $\mathbf{b}_{n, p}(x)$ & $\widehat{\mathbf{p}}_{\lambda}(x)$ & $\mathbf{p}_{\lambda}(x)$ & $\left|\mathbf{b}_{n, p}(x)-\widehat{\mathbf{p}}_{\lambda}(x)\right|$ & $\left|\mathbf{b}_{n, p}(x)-\mathbf{p}_{\lambda}(x)\right|$ \\
\hline 0 & 0.00665397 & 0.00665425 & 0.00673795 & 0.00000028 & 0.00008398 \\
1 & 0.03343703 & 0.03343843 & 0.03368973 & 0.00000140 & 0.00025271 \\
2 & 0.08392862 & 0.08393221 & 0.08422434 & 0.00000359 & 0.00029572 \\
3 & 0.14030279 & 0.14030964 & 0.14037390 & 0.00000685 & 0.00007111 \\
4 & 0.17573100 & 0.17574274 & 0.17546737 & 0.00001173 & 0.00026363 \\
5 & 0.17590762 & 0.17592636 & 0.17546737 & 0.00001874 & 0.00044025 \\
6 & 0.14658968 & 0.14661618 & 0.14622281 & 0.00002650 & 0.00036687 \\
7 & 0.10460168 & 0.10463358 & 0.10444486 & 0.00003190 & 0.00015682 \\
8 & 0.06524464 & 0.06527707 & 0.06527804 & 0.00003243 & 0.00003340 \\
9 & 0.03613774 & 0.03616583 & 0.03626558 & 0.00002809 & 0.00012784 \\
10 & 0.01799623 & 0.01801726 & 0.01813279 & 0.00002103 & 0.00013656 \\
11 & 0.00813900 & 0.00815280 & 0.00824218 & 0.00001380 & 0.00010318 \\
12 & 0.00337080 & 0.00337884 & 0.00343424 & 0.00000804 & 0.00006344 \\
13 & 0.00128734 & 0.00129155 & 0.00132086 & 0.00000421 & 0.00003352 \\
14 & 0.00045607 & 0.00045806 & 0.00047174 & 0.00000199 & 0.00001567 \\
15 & 0.00015065 & 0.00015151 & 0.00015725 & 0.00000086 & 0.00000660 \\
16 & 0.00004660 & 0.00004695 & 0.00004914 & 0.00000034 & 0.00000253 \\
17 & 0.00001356 & 0.00001368 & 0.00001445 & 0.00000013 & 0.00000090 \\
18 & 0.00000372 & 0.00000376 & 0.00000401 & 0.00000004 & 0.00000029 \\
19 & 0.00000097 & 0.00000098 & 0.00000106 & 0.00000001 & 0.00000009 \\
20 & 0.00000024 & 0.00000024 & 0.00000026 & 0.00000000 & 0.00000003 \\
21 & 0.00000006 & 0.00000006 & 0.00000006 & 0.00000000 & 0.00000001 \\
22 & 0.00000001 & 0.00000001 & 0.00000001 & 0.00000000 & 0.00000000 \\
\hline
\end{tabular}

The improved approximation is better than the Poisson approximation.

\section{Conclusion}

The result of this study is an improved Poisson distribution with mean $\lambda=$ $n p$. It can be used as a good approximation of the binomial distribution with parameters $n$ and $p$ when $n$ is large. Additionally, the improved approximation is better than the Poisson approximation.

\section{References}

[1] T.W. Anderson, S.M. Samuels, Some inequalities among binomial and Poisson probabilities, in Proceedings of the Fifth Berkeley Symposium on Mathematical Statistics and Probability, vol. 1, 1965, pp. 1-12.

[2] S. Antonelli, G. Regoli, On the Poisson-binomial relative error, Statistics \& Probability Letters, 71 (2005) 249-256.

[3] I.W. Burr, Some approximate relations between terms of the hypergeometric, binomial and Poisson distributions, Communications in Statistics, 1 (1973) 293-301. 
[4] W. Feller, An introduction to probability theory and its applications, vol. 1, Wiley, New York, 1968.

[5] D.P. Hu, Y.Q. Cui, A.H. Yin, An improved negative binomial approximation for negative hypergeometric distribution, Applied Mechanics and Materials, 427-429 (2013), 25492553.

[6] N.L. Johnson, S. Kotz, A.W. Kemp, Univariate Discrete Distributions, 3rd ed., Wiley, New York, 2005.

[7] K. Teerapabolarn, A bound on the Poisson-binomial relative error, Statistical Methodology, 4 (2007) 407-415.

[8] K. Teerapabolarn, A Poisson-binomial relative error uniform bound, Statistical Methodology, 7 (2010) 69-76.

[9] K. Teerapabolarn, An improvement of bound on the Poisson-binomial relative error, International Journal of Pure and Applied Mathematics, 80 (2012) 711-719.

[10] K. Teerapabolarn, A new non-uniform bound on the Poisson-binomial relative error, International Journal of Pure and Applied Mathematics, 86 (2013) 35-42. 
